\title{
Robust data transmission with eddy current sensor system
}

\author{
S. Hensel, C. Hasberg \& R. Rütters \\ Institut für Mess- und Regelungstechnik, \\ Universität Karlsruhe(TH), Germany
}

\begin{abstract}
Nowadays railway supervision systems use information carriers installed along the track to define the train position or to provide information about the track as for example maximum velocities. Current data transmission devices are electronic systems, which are expensive to install and maintain. This paper proposes a novel approach to use an eddy current sensor system, mounted on trains for speed measurements, to receive data coded in a metal bar on the track. The transmission is realized with a quadrature amplitude modulation in combination with the given eddy current sensor system to show a cost-efficient and robust alternative to common railway supervision systems.
\end{abstract}

Keywords: eddy current sensor system, QAM, railway disposition.

\section{Introduction}

Actual railway disposition and supervision systems are capable of ensuring a safe railway transportation of goods and men. These systems use information carriers placed along the track to define the position of the train or to provide information about the track as for example maximum velocities. Currently, these data transmission devices are electronic systems that are activated by the passing train to transmit information. Drawbacks of these systems are the high installation and maintenance costs. Moreover it is necessary to equip the train with a special receiver only capable to work with specially designed encoders.

This paper describes an elegant way to use an eddy current sensor system, already mounted on a train bogie to measure speed, by systematically manipulating the electromagnetic properties within the measurement range of the sensor system. The eddy current sensor system was originally developed at the depart- 
ment for measurement and control at the University of Karlsruhe [1]. The application range of the sensor system includes contactless velocity measurement based on correlation methods and classification of characteristic signal patterns that are recorded when the sensor passes special track components, e.g. turnouts (for further information see [2] and [3]).

The remainder of this paper is organized as follows. Section 2 gives an overview on the system and reviews important properties, that make the quadrature amplitude modulation (QAM) a robust method to transmit information. Based on a systematic identification of parameters of the data transmission channel a system concept to realize a robust data transmission is introduced in Section 3. It describes the detection of characteristic signal patterns by using orthogonal correlation, while the correlation frequency is adapted to the actual velocity of the train. Afterwards, Section 4 depicts the experimental setup to test the system in laboratory conditions in detail. Section 5 presents the experimental results and a summary of the proposed approach.

\section{System overview}

An eddy current sensor system (ECS) in combination with a metal bar is used as hardware to realize the data transmission between encoder and train. The bar is placed parallel to the rails and encodes the information by changing the amplitude and phase of the signal received of the sensor. This principle can be viewed as a barcode. The information coding and decoding employs QAM.

\subsection{Quadrature amplitude modulation}

Prominent digital modulation techniques include amplitude, phase, and frequency modulation. For the QAM, the following assumptions are made. The wanted signal is given by $u(t)$, defined in Equation 1, where $A(t)$ is the amplitude and $\varphi(t)$ the phase. This can be expressed as:

$$
\begin{aligned}
u(t) & =A(t) \cdot e^{\jmath \varphi(t)}=A(t) \cos \varphi(t)+J A(t) \sin \varphi(t) \\
& =I(t)+J Q(t)
\end{aligned}
$$

where $I(t)$ and $Q(t)$ are called the inphase and quadrature components of the wanted signal. The given information, e.g. binary coded, is then transformed into a sequence of amplitudes and phases $I(n)$ and $Q(n)$. This transformation is done by a so called mapper, which allocates the given symbols with the corresponding amplitude and angle in the complex space according to the given scheme. A time signal is won by sampling the signal at the sampling instances $n \cdot T$ with $\delta$ impulses and a following low pass filtering. Afterwards, the wanted signal is modulated on a carrier frequency $\omega_{c}=2 \pi f_{c}$. The inphase component $I(t)$ is therefore multiplied with the real part of the carrier signal $\cos \left(\omega_{c} t\right)$ and the quadrature component $Q(t)$ is multiplied with the complex part of the carrier signal $\sin \left(\omega_{c} t\right)$, leading to the 


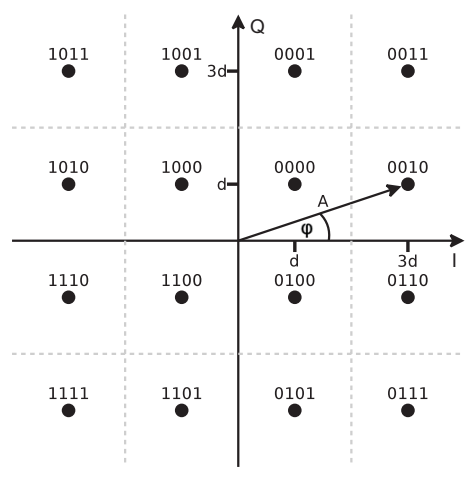

Figure 1: A 16 QAM square or Type III QAM constellation, showing the phasor points and the amplitude $A=\sqrt{I^{2}+Q^{2}}$ and phase $\varphi$.

transmitted signal $s(t)$ :

$$
s(t)=\operatorname{Re}\left\{u(t) \cdot e^{j \omega_{c} t}\right\}=I(t) \cdot \cos \left(w_{c} t\right)-Q(t) \cdot \sin \left(w_{c} t\right)
$$

In this application a square QAM is chosen for coding. The principle is illustrated in a constellation diagram of phasor points. A phasor point represents a data word, where each word encodes 4 bit. For the combination with the ECS system a 16 points square, or the so called 16 points Type III QAM, constellation is used and depicted more detailed in Figure 1 (see [4] for further details). The inphase and quadrature components $I(t)$ and $Q(t)$ are used for the modulation process, coding the data words into the QAM plane. Each phasor point is defined by its own amplitude $A$ and phase $\varphi$. Figure 1 shows the decision boundaries, indicating the shift of one data word to another, marked by the gray dotted lines. The arrangement of the phasor points ensures robustness against noise. This Gray coding [5] of the signal space in combination with a code, that has a small hamming distance represents a robust coding scheme for the given sensor system.

\subsection{Eddy current sensor}

The eddy current sensor system consists of two identical sensor devices. Each one containing a transceiver coil and two pick up coils to receive the signal. The two sensors are placed in a row within a housing, which is mounted on a train bogie approximately $0.1 \mathrm{~m}$ above the rail head. Figure 2(a) shows the working principle of a single sensor used in the sensor system. The sensor sends out a magnetic field, which induces eddy currents in a metallic material within the range of the transceiver coil $E$. These currents induce an antipode magnetic field, which then induces voltage in the two pick up coils ( $P 1$ and $P 2)$. These coils are interconnected differentially, and provide the output signal $s(t)=u_{P 1}(t)-u_{P 2}(t)$. Therefore the receivers are only capable to detect inhomogeneities in the magnetic resistance along the track caused by rail clamps, turnouts or irregularities, e.g. 


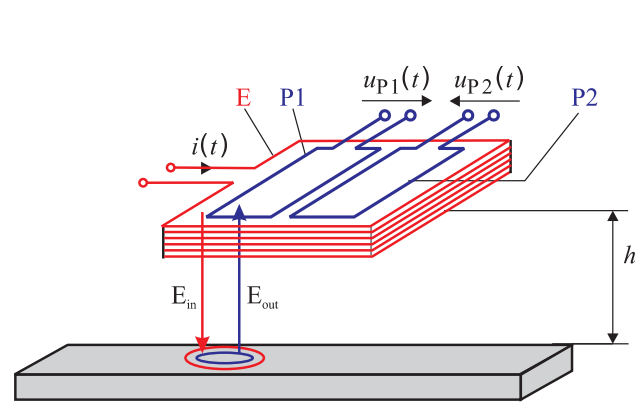

(a)

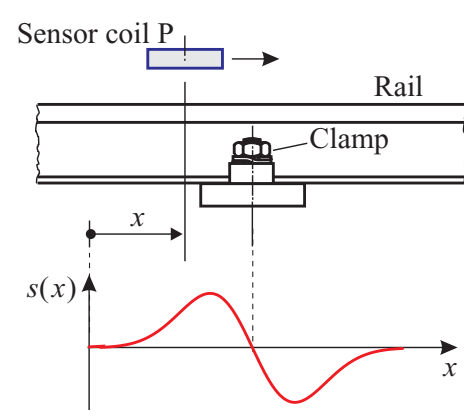

(b)

Figure 2: (a) Working principle of ECS system (b) Signal of a differential sensor moved over a metal sheet.

cracks, of the rail [2]. To use the sensor system to determine the train velocity, the principle of a closed-loop correlator is used. Both sensors are scanning the magnetic resistance to the rail, separated by a known distance $l$. In this arrangement a cross correlation is applied to the signals. This function determines the time difference $T$ of the two sensor signals, represented at the position of the maximum. The train velocity is then readily obtained by $v=l / T$. This measurement is widely unsusceptible to environmental perturbations and other interferences. Figure 2(b) shows the shape of the signal won by driving over a metal sheet $M$ with one of the differential sensors.

\section{System implementation}

This chapter describes how the QAM is adjusted the ECS. The QAM parameters phase and amplitude have to be specified, as well as the frequency of the carrier signal, depending on the actual train velocity.

\subsection{Signal model}

Figure 2(b) shows a typical ECS signal, acquired by driving over a metal plate with one of the differential sensors. The shape of this signal results from the low pass characteristic of the sensor. Taken this into account, an ideal model can be derived, the shape of which is shown in Figure 3. This signal model can be applied, if the length $l_{M}$ of the metal plate is equal to the width of the pick-up coil $l_{S}$ and a constant speed $v$ is assumed. This is resulting in the sawtooth shape, which is separated in three domains: The areas I, II, III, each covering the same time span $\Delta t$, if a constant speed $v$ is assumed. Domain I represents the intrusion of the first pick-up coil in the field of the metal sheet. Domain II the equilibrium of both 


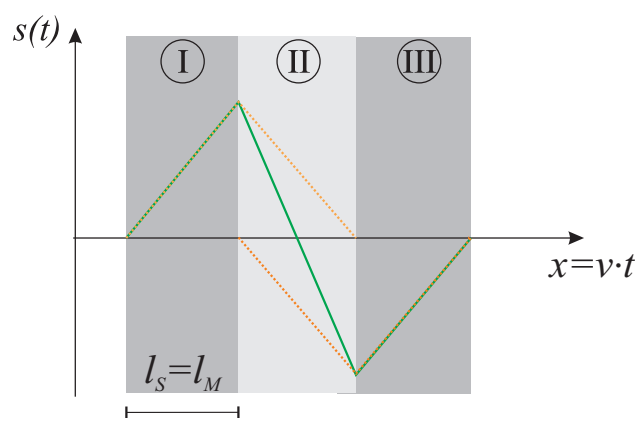

Figure 3: Idealized ECS signal of each pick-up coil (dotted) and resulting signal $s(t)$ (solid).

coils and finally domain III the exit of the second pick-up coil. The signal of each coil and the resulting difference $s(t)$ is depicted. Given these parameters the signal frequencies $f_{I}, f_{I I}, f_{I I I}$ (according to the areas) can be defined as:

$$
2 f_{I}=2 f_{I I I}=f_{I I}
$$

The two frequencies define two different triangles, which can be expressed by the fourier series of a triangle function, given by

$$
\hat{s}(t)=\frac{8}{\pi^{2}} \sum_{k=0}^{N} \frac{\cos ((2 k+1) t)}{(2 k+1)}
$$

Because of the mentioned low pass characteristics, $N$ can be chosen small. With the use of Equation 4, the signal $\tilde{s}$ later used for detection is approximately $\hat{s}$, if only the first element of the series is taken into account for signal detection like shown in Section 3.2.

\subsection{Signal detection}

An orthogonal correlation can be used to identify the signal and its parameters under the assumptions of the model made in Section 3.1. This approach is widely spread and is mostly used for identification of linear systems and data transmission [6]. The orthogonal correlator receives an input signal $s(t)$, in the given application the sensor signal, which is split into two paths and multiplied with a sine and a cosine with the correlator frequency $f_{T}$. The sine and cosine build an orthogonal basis scaled to one for each correlation length:

$$
\begin{aligned}
\Phi_{I}(t) & =\frac{2}{T} \cos 2 \pi f_{T} t \\
\Phi_{Q}(t) & =\frac{2}{T} \sin 2 \pi f_{T} t
\end{aligned}
$$


The sines are multiplied and integrated to build a correlation with $s(t)$. Because of the orthogonal attribute is it possible to define the first path as the inphase component $I(t)$ and the second as the quadrature component $Q(t)$, afterwards treated analogue to the QAM (see Section 2.1). Finally the output is converted into an amplitude $A(t)$ and a phase-angle $\varphi(t)$, accordingly to Equations (5)-(9).

$$
\begin{aligned}
I(t) & =\int_{0}^{T} s(t) \Phi_{I}(t) d t \\
Q(t) & =\int_{0}^{T} s(t) \Phi_{Q}(t) d t \\
I(t)+J Q(t) & =A(t) e^{j \varphi(t)}
\end{aligned}
$$

Both, the inphase and quadrature components, are influenced by the correlator frequency $f_{T}$. This affects the first output, the amplitude in a way that it is smaller and decaying faster if the higher frequency $f_{I I}$ is chosen as correlator frequency (see [7]). The phase angle $\varphi(t)$ as the second output of the correlator is determined by the position of a metal sheet relative to a starting point. In Figure 4 an example for the phase shift in dependency of a metal sheet with the length $l_{M}$ is shown. The phase is now expressed as a function of the displacement $x_{d}$ of the metal sheet. The global relation is given with

$$
\frac{\varphi_{d}}{2 \pi}=\frac{x_{d}}{\lambda}
$$

where $\lambda$ is the wavelength of the sine depending on the correlator frequency $\frac{v}{f_{T}}=$ $\lambda, \varphi_{d}$ the desired phase and $x_{d}$ the displacement of the metal sheet. This relation has a direct anti proportional impact on the information carrier length. Therefore the argument $f_{T}$ of $\Phi_{I}(t)$ and $\Phi_{Q}(t)$, has to be set under consideration of the previous assumptions. To assure the shortest carrier length possible, a compromise of a slightly lower amplitude is accepted and the frequency is set to $f_{T}=f_{I I}$. The integration length $T$ of the correlator is set to $T=\frac{1}{f_{T}}$.

\subsection{Information carrier}

The aim of the described application is to transmit a signal with a maximum signal length of 16 bit. Therefore, the information properties are set assuming the use of a 16 QAM: Four data words, each containing four bit are sent. In order to code each data word three different signal amplitudes and twelve phase angles have to be defined. The calculation principle is depicted in Figure 5.

\subsubsection{Amplitude}

Equations (12)-(14) define the amplitudes as a function of the distance $d$ in signal space. The amplitudes $A_{2}$ and $A_{3}$ are expressed in dependency of the maximum amplitude $A_{1}$. This property is used in Section 4.1 to calculate proper heights 
for the encoders. In [2] the dependency of the signal amplitude given the sensor height $z$ over the rail head is derived in detail. An exponential approximation with experimental measurements leads to the following equation describing the amplitude in dependency of the distance $h$ from the sensor base to an object:

$$
A(h)=A_{\max } \cdot e^{\frac{-0.0316 \mathrm{~h}}{\mathrm{~mm}}}
$$

Given this equation, the amplitudes $A_{1}, A_{2}$ and $A_{3}$ can be derived like shown in Figure 6 . The maximum amplitude $A_{1}$ is determined by the minimum distance $h_{\min }$ between the sensor and the railhead and can be adjusted by amplification. This realization of the amplitude coding is very robust to bogie movements and height changes, due to the relative definition of the amplitudes.

\subsubsection{Phase angle}

To encode the correct phase the first three values are calculated with the tangens of $Q(t)$ and $I(t)$. These phase angles are then used to predict the other angles by

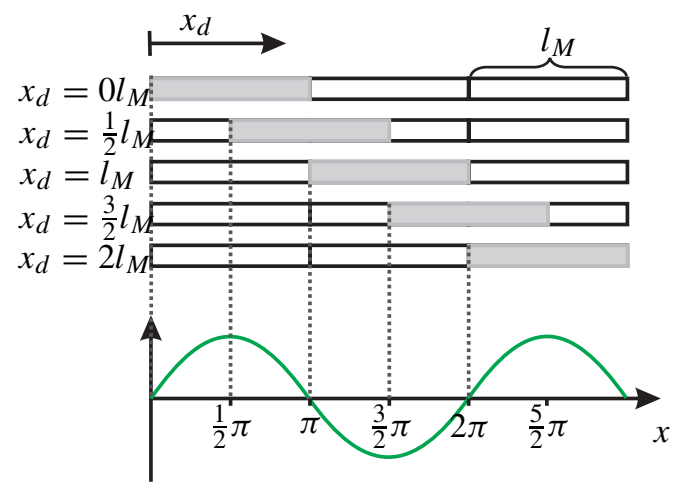

Figure 4: Phase related to displacement $x_{d}$ of a metal sheet with the length $l_{M}$.

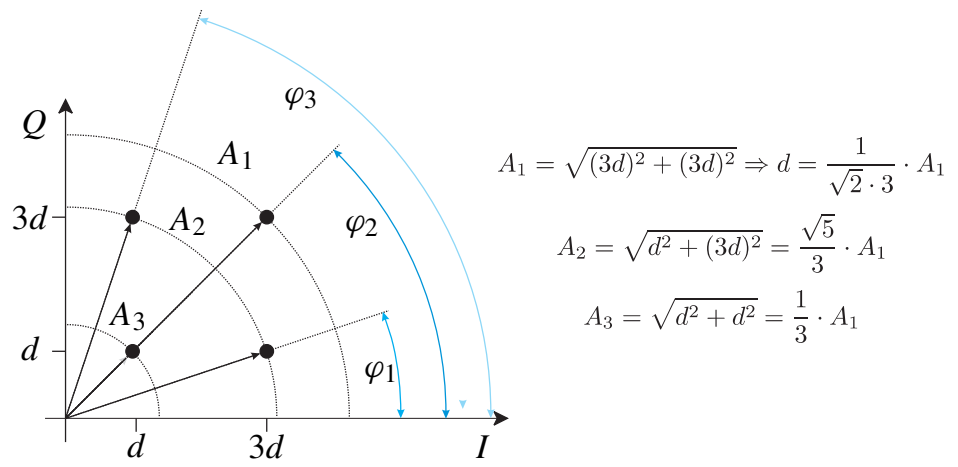

Figure 5: 16 QAM signal space cut out for exemplarily parameter calculation. 


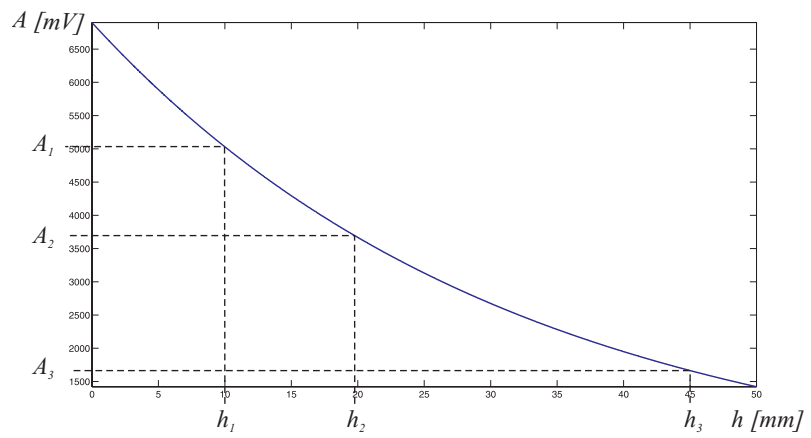

Figure 6: Signal amplitude $A$ as function of the distance from sensor to encoder $h$.

adding 90 in each quadrant.

\subsubsection{Information carrier arrangement}

To realize the transmitter parameters mentioned above, ideal reflecting encoders are assumed. As depicted in Figure 3 the signal is subdivided into three areas of the same length $l_{M}$. The assumption that the encoder length equals $l_{M}$, leeds to a distance of $3 l_{M}$ between the points where the sheet enters and leaves the sphere of the sensor's sensivity. Therefore the distance between two metal sheets has to be at least $2 l_{M}$, if a signal code with no inter symbol interference is to be used.

An additional start word with the length $3 l_{M}$ is used to adjust the correlator phase to the signal phase to zero. Afterwards, the simplest way to code the signal information would be to reserve $5 l_{M}$ for each word ( $2 l_{M}$ for spacing and $3 l_{M}$ for phase manipulation). A drawback of this method is the phase drift, due to a correlator timing slightly different from the ideal carrier timing because of noise and errors in the velocity measurement, which will grow with the length of the carrier.

A more efficient and robust way of coding is outlined in the sequel. The basic principle is a reset of the phase counter to "0" after each transmitted word. Thus, each data word can be treated as if a new measurement is started. This is realized if the length of one word is not assumed to be constant, but in dependency of the position of the encoding metal sheet. After each encoder sheet a free space of $2 l_{M}$ is introduced between the next sheet. This leads to a reduction of the phase error and has the advantage of a shorter carrier length. An example of this coding method is shown in Figure 7, coding the following bit sequence "0011 10100010 1001". The calculated phase angles in multiples of $l_{M}$ are outlined in Table 1: The length of this arrangement type is defined by the formula:

$$
l_{\text {total }}=3(n+1)+\sum_{i=1}^{n} x_{d, i} .
$$

The length of the depicted example carrier in multiples of $l_{M}$ is $l_{\text {total }}=20.772 l_{M}$. 
Table 1: Calculated phase angles of the example data sample.

\begin{tabular}{|l|c|c|c|c|}
\hline $\mathrm{n}$ & 1 & 2 & 3 & 4 \\
\hline \hline Dataword & 0011 & 1010 & 0010 & 1001 \\
\hline Phase & $\varphi_{2}$ & $\varphi_{6}$ & $\varphi_{1}$ & $\varphi_{4}$ \\
\hline Phase [rad] & $\frac{1}{4} \pi$ & $0.8978 \pi$ & $0.1024 \pi$ & $0.6022 \pi$ \\
\hline Phase-Shift & $\pi$ & $\pi$ & $\pi$ & $\pi$ \\
\hline FinalPhase & $\frac{5}{4} \pi$ & $1.8978 \pi$ & $1.1024 \pi$ & $1.6022 \pi$ \\
\hline$x_{d, n}$ & $1.25 l_{M}$ & $1.8978 l_{M}$ & $1.1024 l_{M}$ & $1.6022 l_{M}$ \\
\hline
\end{tabular}

This saves $9.6 \%$ relative to the length of the word arrangement first explained. The minimal possible length for the carrier is the bit string "0101 010101010101 ", coded with $15.512 l_{M}$. This would result in a relative saving of $32.6 \%$.

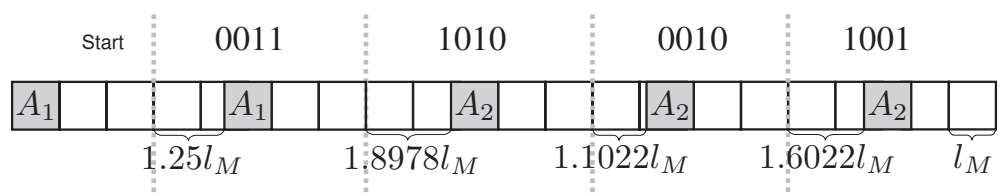

Figure 7: Example carrier arrangement for data word coding.

For an uniformly distributed symbol occurrence the average length of one symbol is given by $4 l_{M}$, for 16 bit this is an average carrier length of $19 l_{M}$.

\section{Experimental setup and results}

The experimental verification of the presented models is done with a laboratory test stand with a sufficient length to place the information carrier. The eddy current sensor is driven by a manually controlled asynchronous motor, the signal carrier is installed directly below the sensor but without a rail. The speed measurement, necessary to adjust the correlator frequency, is measured with light carriers. The test stand is depicted in Figure 8. The evaluation unit, build in cooperation with Bombardier RTCS Stockholm is used to preprocess the signals and sends them via RS 422 to a host PC.

\subsection{Information carrier setup}

The test carrier is subdivided in two parts. In the beginning a reference block represents the start word. The positioning of the block is set to have a distance of 


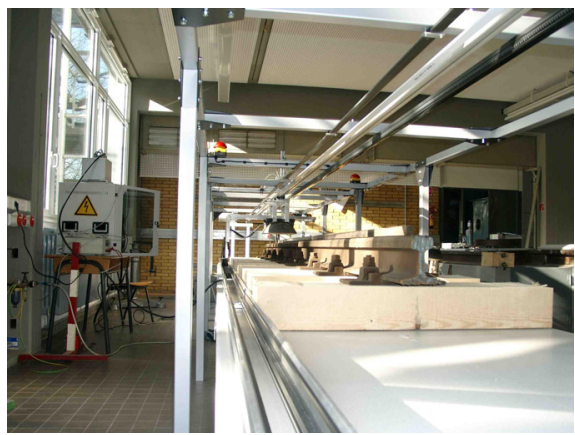

Figure 8: The ECS test stand of the department of measurement and control at the University of Karlsruhe.
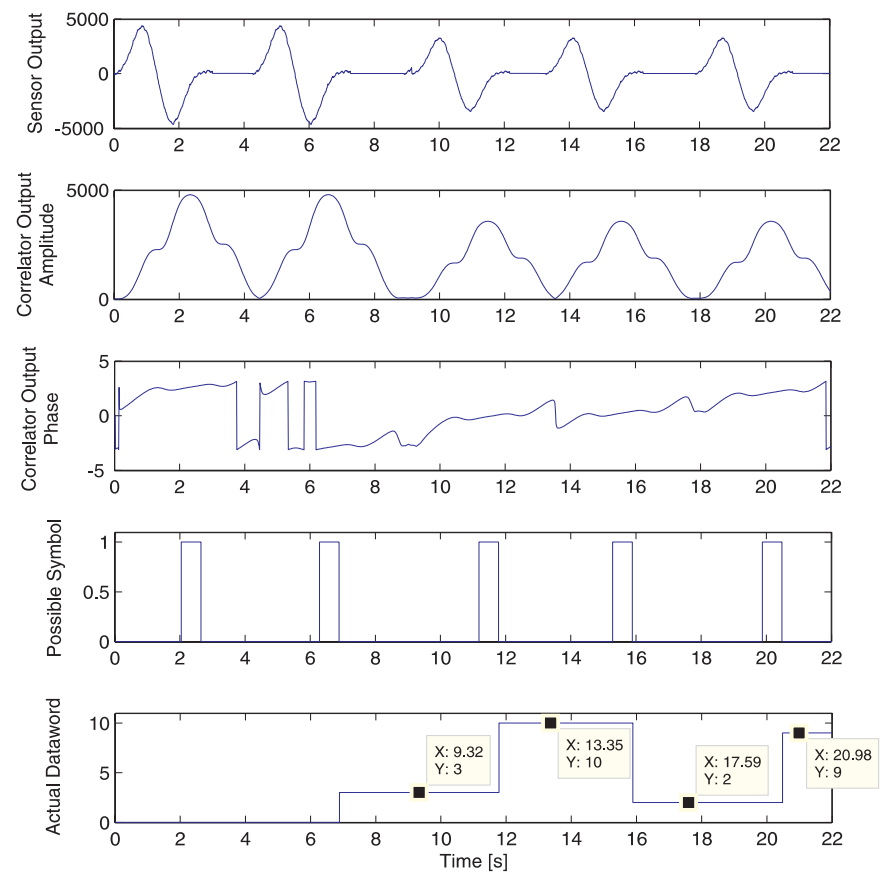

Figure 9: Decoding results.

$0.1 \mathrm{~m}$ to the sensor to induce a maximum amplitude in the ECS system. Based on the geometric properties of the sensor, the optimal length of the encoding sheets is $l_{M}=140 \mathrm{~mm}$. On the top of the block an aluminium sheet with length $l_{M}$ is embedded. The second part, containing the relevant information, starts after the calibration word and represents the data of 4 byte. PVC baseplates are used to get the blocks in position. Each baseplate has a length of $3 l_{M}=420 \mathrm{~mm}$ and contains several boreholes for a flexible placement of the block. After setting the phase, the 
amplitude is coded by the distance to the sensor and therefore the block height. The height can be set as described in Section 3.3.1. For a 16 square QAM, the block heights in the experimental set-up are set to $44.79 \mathrm{~mm}, 35.48 \mathrm{~mm}$ and $10 \mathrm{~mm}$. The total length of the carrier for the given example is $2.91 \mathrm{~m}$. The results are shown in Figure 9. The metal sheets in the test setup are made of aluminium. Because the sensor amplitude is a function of the material conductivity they are inducing a clearer signal than those made of iron or steel. Another benefit is the use of a PVC body and the absence of rails and infrastructure parts as disturbances. Figure 9 depicts in detail the measured signal, correlator amplitude, correlator phase and the corresponding analogue values to the data words 3, 10, 2 and 9.

\section{Conclusions}

This paper proposes data transmission with an eddy current sensor system, originally designed to measure train speed. It does not necessitate any electric or moving components on the track and thus offers itself for cheap and almost maintenance-free data transmission.

The experimental setup described in the previous sections provides excellent results, employing aluminium encoders, thorough information carrier placement and a missing rail. The correlator frequency is adjusted from the exact speed measurement by the ECS itself.

For further research and implementation on tracks additional disturbances have to be taken in account. At first, rails have to be added beside the information carrier. Second, the carrier must be replaced with an iron body for the sake of low costs and to prevent demolition or thievery. The varying speed during train operation has to be taken into account as well as failures in the speed measurements, which lead to a slightly shifted correlator frequency. The sensor has proven extremely robust to numerous real world challenges, such as bogie vibration or hard weather influences, during extensive tests conducted for turnout classification and speed measurement (see [8] and [9]). The length of the carrier, which is actually varying between $2.17 \mathrm{~m}$ and $3.16 \mathrm{~m}$ can be shortened by a different coding of the information. An even more robust signal decoding can be achieved with an alternative signal space in the QAM, e.g. a star alignment. Further improvements will result out of the redundant setup of the ECS system. Because two sensors are set in a row, reception of the transmitted information is redundant.

\section{References}

[1] Engelberg, T., Speed measurement of rail vehicles using shielded eddy current sensors - field-test 3/00 in munich. Technical Report AV 05/00, Institut für Mess- und Regelungstechnik, Universität Karlsruhe (TH), 2000.

[2] Engelberg, T., Geschwindigkeitsmessung von Schienenfahrzeugen mit Wirbelstrom-Sensoren, volume 8, Nr. 896. VDI: Düsseldorf, 2001. ISBN: 318-389608-7. 
[3] Geistler, A. \& Böhringer, F., Detection and classification of turnouts using eddy current sensors. Computers in Railways IX, eds. J. Allan, R. Hill, C. Brebbia, G. Sciutto \& S. Sone, WIT Press: Southampton, pp. 467-476, 2004.

[4] Hanzo, L., Quadrature amplitude modulation. Wiley, 2004.

[5] Shanmugam, K.S., Digitaland Analog Communication Systems. Wiley, 1979.

[6] Jondral, F., Nachrichtensysteme. Schlembach, 2nd edition, 2006.

[7] Rtters, R., Entwicklung einer passiven Balise zur Datenbertragung im Schienenverkehr. Master's thesis, University of Karlsruhe (TH), Institute for Measurement and Control Theory, 2007.

[8] Hensel, S. \& Geistler, A., Laboratory and field tests of a new eddy current sensor system. Technical report, Institut für Mess- und Regelungstechnik, Universität Karlsruhe (TH), 2006.

[9] Hensel, S., Summary of regina winter test in västerås. Technical report, Institut für Mess- und Regelungstechnik, Universität Karlsruhe (TH), 2007. 